\title{
Improvement of Nonlocal Peierls-Nabarro Models
}

\author{
Guisen Liu ${ }^{\mathrm{a}}$, Xi Cheng ${ }^{\mathrm{a}, \mathrm{b}}$, Jian Wang ${ }^{\mathrm{c}}$, Kaiguo Chen ${ }^{\mathrm{d}}$, Yao Shen ${ }^{\mathrm{a}, *}$
}

\begin{abstract}
We review the major efforts that improve the accuracy of Peierls-Nabarro (PN) model in predicting core structure and Peierls stress, and recognize that the nonlocal atomic interactions in the core region should be accounted for in calculating the dislocation energy. Although some efforts have been devoted to taking the nonlocal interaction into account, further improvement is needed to simplify the computational complexity and resolve the inconsistency between the continuum model and the discrete nature of the lattice. Here we developed a two-dimensional (2D) and a three-dimensional (3D) nonlocal semi-discrete variational Peierls-Nabarro (SVPN) models by incorporating the nonlocal atomic interactions into the semi-discrete variational Peierls framework. The nonlocal SVPN models are applied to dislocations with extended core in copper and compact core in iron. Molecular dynamics simulations are performed to validate the model predictions. We found that the nonlocal SVPN model (both 2D and 3D) significantly improves the prediction accuracy for dislocation core structure and Peierls stress. Moreover, the results show that the 2D and 3D models give similar predictions of the Peierls stress and dislocation core structure, when the atomic relaxation in the normal direction is allowed in the $2 \mathrm{D}$ model to describe the interatomic interactions in the slip plane.
\end{abstract}

Keywords: Dislocation; Nonlocality; Peierls stress; Peierls-Nabarro model; Molecular dynamics 


\section{Introduction}

Crystal plasticity is accommodated by dislocation slips and localized shear transformations (such as twinning and phase transformation) that are accomplished by the movement twinning dislocations/disconnections or steps. The mobility of these linear defects to a great extent determines mechanical properties of materials. Therefore, accurate prediction of Peierls stress for a dislocation (the minimum external stress to move a straight dislocation) is vital to understand the mobility of the dislocation. Much effort has been devoted to measure and estimate the Peierls stress associated with dislocations by using theory, modeling, and experiments [1-4]. Molecular statics/dynamics simulations that are capably of calculating atomic interactions in full three dimensions were extensively used to study dislocation behaviors including nucleation, motion, and reactions [5-7]. However, it is limited for complex system because of the unavailability of the empirical potentials for molecular statics/dynamics simulations. Atomistic calculations using first principles density function theory have no dependence on potentials while have the limited simulation volumes. Thus it is rarely used to study the motion of dislocations.

Continuum elasticity theory well describes the long-range elastic strain of a dislocation for length scales beyond a few lattice spacing, but it breaks down in the region surrounding the dislocation center (referred to as the dislocation core). The early crystallographic model developed by Peierls and Nabarro (PN) $[8,9]$ (referred to as original PN model) was the first one to describe crystallographic character of a dislocation core in the frame of continuum elasticity theory based on the assumption of one dimensional sinusoidal law for atomic interactions across the slip plane, and its analytical solution gave the core structure and Peierls stress for the first time. This method is naturally associated to the inconsistency between the continuum model and the discrete nature of the lattice $[10,11]$. There has been a great deal of interest in describing accurately the core structure of a dislocation at the atomic scale because the structure and properties of dislocation core control the mobility of the dislocation, which accounts for the intrinsic ductility or brittleness of solids. To capture the core structure of a dislocation, several improvements [11-15] have been proposed in past 
decades. Vitek [12, 13] proposed a more physically realistic way to describe atomic interactions by replacing the sinusoidal force with the gradient of the $\gamma$-surface (the model is thus referred to as the $\gamma$-PN model), and from the maximum slope of which, the Peierls stress of dislocations with both narrow and wide core can be directly estimated $[16,17]$. Schoeck $[14,15]$ further generalized the $\gamma$-PN model to a two dimensional one. The $\gamma$-PN models have been frequently adopted to investigate dislocation properties [18-21] for its efficiency in obtaining the two dimensional dislocation core. Inconsistency between the continuum and discrete treatment of the dislocation in the $\gamma$-PN model was overcome by the semi-discrete variational Peierls framework (referred to as SVPN model) [11]. In SVPN model, discrete nature of the lattice was accounted for in calculating the dislocation energy, and the dislocation profile was allowed to fully relax in three dimensions as the dislocation moves in lattice under the external applied stress [11].

All these improvements were made within the locality assumption, i.e., the misfit energy in the region of $\mathrm{dx}$ depends only on the $\gamma$-surface at that local atomic site, which was implied in that the $\gamma$-surface was calculated using a uniform disregistry vector. However, the large gradient in the dislocation profile has been demonstrated using atomistic simulations but not incorporated into PN models. Schoeck [22] and Miller et al [23] modified the misfit energy in the slip plane by considering the large displacement gradient effects in the core region in order to account for the nonlocal effects. Their applications were limited due to the complicated nonlocal mathematical formulation or the difficulty in assessing the nonlocal parameters. In this work, we developed an improved two-dimensional (2D) nonlocal SVPN model by incorporating the nonlocal atomic interactions into the SVPN model, wherein the form of the nonlocal interaction energy term was inspired from the nonlocal kernel derived by Miller et. al. [23] but extended to two dimensions in a simpler form, and the nonlocal coefficient is computed directly from the dislocation core structure. Moreover, we extended the improved 2D nonlocal SVPN model to a three-dimensional (3D) one by explicitly introducing the vertical component of the disregistry vector in the dislocation energy. Finally, we tested our 2D and 3D nonlocal SVPN models for dislocations in iron and copper, by 
comparing predictions for the core structure and Peierls stress with MD simulations.

\section{Typical PN models}

\subsection{Local PN models}

In the original PN model $[8,9,24]$, dislocation energy is composed of two parts, the elastic energy stored in the two half continuum linear elastic solid separated by the dislocation slip plane, and the atomic misfit energy in the slip plane to account for the non-linear interatomic interaction, which is confined in the slip plane. The dislocation profile or the disregistry, $\delta(x)$ (relative displacement of the atom pairs across the shear plane), should minimize the total energy. Correspondingly, the total energy is a functional of the dislocation profile,

$$
E[\delta(x)]=E_{\text {elastic }}+E_{\text {misfit }}=K \iint \frac{d \delta\left(x^{\prime}\right)}{d x^{\prime}} \frac{\delta(x)}{x-x^{\prime}} d x d x^{\prime}+\int \gamma[\delta(x)] d x
$$

where the two terms on the right hand are the elastic energy and the misfit energy respectively. $K$ depends on the dislocation type, and equals to $\mu /(4 \pi)$ for a screw dislocation and $\mu /(4(1-v) \pi)$ for an edge dislocation assuming isotropic elasticity, where $\mu$ is the shear modulus and $v$ is the Poisson's ratio. $\gamma[\delta(x)]$ is the misfit potential to describe the interatomic interaction across the slip plane, gradient of which is the atomic restoring force. The dislocation profile is obtained by minimizing the total energy. Then Peierls stress is determined as the maximum slope of the misfit energy variation as the dislocation profile is rigidly translated in crystal for one lattice period.

Three major simplifications were made in the original PN model. (i) One dimensional sinusoidal form for the periodical misfit potential $\gamma[\delta(x)]$ was assumed to describe the interatomic interactions. (ii) Inconsistency exists in calculating the misfit energy, which was obtained by continuously integrating the $\gamma[\delta(x)]$ in Eq. (1) to obtain the dislocation profile $\delta(x)$, but to determined Peierls stress it was obtained by discretely summing up $\gamma\left[\delta\left(x_{i}\right)\right]$ at atomic sites $x_{i}$. (iii) Dislocation profile was rigidly translated in the lattice without relaxation in determining the Peierls stress.

To better describe the interatomic interactions, the concept of the $\gamma$-surface (the 
generalized stacking fault energy (GSF energy) surface firstly proposed by Vitek [12, 13]) was used. Joos et al $[16,17]$ firstly modified the magnitude of the sinusoidal misfit potential according to the maximum gradient of the $\gamma$-surface. Later, Schoeck $[14,15]$ directly replaced the sinusoidal misfit potential in Eq. (1) with the $\gamma$-surface, and the PN model was thus generalized to a $2 \mathrm{D}$ one (referred to as $\gamma$-PN model), the dislocation energy is written as,

$$
E[\vec{\delta}(x)]=E_{\text {elassic }}+E_{\text {misfit }}=\iint \frac{d \vec{\delta}\left(x^{\prime}\right)}{d x^{\prime}} \frac{H}{x-x^{\prime}} \vec{\delta}(x) d x d x^{\prime}+\int \gamma[\vec{\delta}(x)] d x
$$

where the dislocation profile $\vec{\delta}(x)$ is a 2D vector spreading in the slip plane. The symmetric Stroh tensor $H$ in the elastic energy term can account for the elastic anisotropy according to anisotropic elastic theory $[25,26]$. The $2 \mathrm{D}$ disregistry profile is solved by variational methods. Peierls stress is calculated based on the variation of the misfit energy (by discretely summing the GSF energy $\gamma\left[\delta\left(x_{i}\right)\right]$ at atomic sites $x_{i}$ instead of continually integrating the GSF energy) with respect to the rigid translation of the dislocation profile in lattice. Due to the availability of $\gamma$-surface from first principles calculations, the $\gamma$-PN model has been widely applied to investigate dislocation properties such as dislocation dissociation, grain boundary dislocations and even dislocation properties under high pressure [18-21].

The inconsistency between the discrete nature of the lattice and the continuum treatment of the $2 \mathrm{D}$ disregistry vector in integrating the dislocation energy, is conquered by SVPN model [11]. The elastic energy and misfit energy in SVPN model were calculated by linearly interpolating the dislocation profile according to the position of the atomic rows $x_{i}$. By doing so, discrete nature of the lattice was accounted for. Moreover, the dislocation profile is treated as a 3D disregistry vector (two in-plane components plus the normal component) to describe the dislocation core. Therefore, the dislocation profile $\vec{\delta}(x)$ is discretized as $\delta_{k}\left(x_{i}\right), k=1,2,3$ denoting the dislocation profile along $x$-, $y$ - and $z$ - direction respectively, where $y$ is the normal direction. The total energy is thus rewritten as

$$
\begin{aligned}
& E\left[\vec{\delta}\left(x_{i}\right)\right]=E_{e l}+E_{m i s f t}-E_{a p p}=\sum_{i, j} \chi_{i j}\left[K_{e}\left(\rho_{1}\left(x_{i}\right) \rho_{1}\left(x_{j}\right)+\rho_{2}\left(x_{i}\right) \rho_{2}\left(x_{j}\right)\right)+K_{s} \rho_{3}\left(x_{i}\right) \rho_{3}\left(x_{j}\right)\right] \\
& +\sum_{i} \gamma\left[\vec{\delta}\left(x_{i}\right)\right] \Delta x-\sum_{i, k} \frac{x_{i+1}^{2}-x_{i}^{2}}{2}\left(\rho_{k}\left(x_{i}\right) \tau_{k}\right)
\end{aligned}
$$


The discrete coefficient $\chi_{i j}$ is

$$
\begin{aligned}
& \chi_{i j}=\frac{3}{2} \phi_{i+1, i} \phi_{j+1, j}+\psi_{i, j}+\psi_{i+1, j+1}-\psi_{i+1, j}-\psi_{i, j+1} \\
& \phi_{i, \mathrm{j}}=x_{i}-x_{j}, \psi_{i, j}=\frac{1}{2} \phi_{i, j}^{2} \ln \left|\phi_{i, j}\right|
\end{aligned}
$$

The first two energy terms correspond to the elastic energy and misfit energy in the discrete forms, similar to those in Eq. (1) for the original PN model. Even for a planar core, the third displacement (normal to the shear plane) is often observed at the center region of the core from atomistic simulations. To mimic the non-uniform distribution of Burgers vectors along the disregistry profile in the three directions, $\rho_{k}\left(x_{i}\right)=\left[\delta_{k}\left(x_{i+1}\right)-\delta_{k}\left(x_{i}\right)\right] /\left(x_{i+1}-x_{i}\right)$, the dislocation density along $k$ direction is introduced in the discrete form. $k=1$ and 3 are in-plane components, i.e., the edge and screw components of the dislocation density. $k=2$ is the out-of-plane component, i.e., the normal component of the dislocation density. The third term is an additional energy term introduced in the SVPN model to account for the interaction of a dislocation with the applied stress $\tau_{k}$, so as to account for the relaxation of the dislocation profiles as a dislocation moves in lattice under applied stress. $\tau_{k}$ (with $\tau_{1}=\tau_{y x}, \tau_{3}=\tau_{y z}$, and $\tau_{2}=$ $\left.\sigma_{y y}\right)$ is the component of the applied stress interacting with its corresponding component of the dislocation density $\rho_{k} . K_{e}=\mu /(4(1-v) \pi)$, and $K_{s}=\mu /(4 \pi)$, which are the same to the energy factors in Eq. (1) under isotropic elasticity. $\Delta x=\left(x_{i+1}-x_{i}\right)$ is the atomic spacing normal to the dislocation line on the shear plane, and $\gamma\left[\vec{\delta}\left(x_{i}\right)\right]$ is the 3D misfit potential, which can be obtained by atomistic simulation [11].

In the SVPN model, dislocation profile at a certain external stress $\tau_{k}$ is obtained by minimizing the energy functional in Eq. (3). Peierls stress is indicated by an instability of the energy function, i.e., an optimal solution for dislocation profile no longer exists as resolved shear stress increases to a critical value [11]. Compared with the traditional way to determine Peierls stress by calculating the maximum slope of the variation of the misfit energy, the new approach is more rigorous, conforming to the expectation that a dislocation can move freely in lattice as long as the resolved applied shear stress exceeds Peierls stress. By directly accounting for the interaction of the applied stress with the dislocation in the energy 
functional, the SVPN model can be applied to predict dislocation dynamical properties such as cross-slip process $[27,28]$.

In the above models, the misfit energy in the glide plane is obtained by either continuum integrating the misfit potential in Eq. (2), or discretely summing up the misfit potential in Eq. (3). By either way, it was assumed that the misfit energy depends on the disregistry profile at a local atomic site, independent of the disregistry profile at its neighboring sites. This locality assumption is caused by the fact that the misfit potential $\gamma[\vec{\delta}]$ is calculated by shearing a uniform misfit vector $\vec{\delta}$ between the two half crystals across the slip plane. In regions far from the dislocation core, the locality assumption is appropriate because the disregistry profile is approximately uniform. But in the core region, the locality assumption is improper because large gradient exists in the disregistry profile due to complicated nonlinear and nonlocal interatomic interactions. Therefore, the aforementioned PN models are referred to as local PN models that neglect the large gradient effect in the core regions.

\subsection{Nonlocal PN models}

To capture the influence of the large gradient of the disregistry profile on the dislocation energy in the core region, Schoeck [22] thus suggested taking average procedure to consider the nonlocal atomic interaction within a characteristic distance $t$, rather than the local treatment by setting $t=0$ and misfit energy only depends on the misfit potential at atomic sites $x_{i}$, the misfit energy of a dislocation is modified as,

$$
E_{\text {misfit }}^{\prime}=\Delta x \sum_{i} \Gamma\left(x_{i}\right)=\Delta x \cdot \sum_{i} \frac{1}{2 t} \int_{-t}^{t} \gamma\left[\vec{\delta}\left(x_{i}+t\right)\right] d t
$$

where $x_{i}$ is the positions of the atom rows, $\Delta x=\left(x_{i+1}-x_{i}\right)$ is the nodal spacing of the atom rows, $\gamma\left[\vec{\delta}\left(x_{i}+t\right)\right]$ is the $2 \mathrm{D}$ misfit potential, and $|t| \leq \Delta x / 2$. However how to assess the characteristic distance $t$ is not clear. Miller et. al. [23] proposed another nonlocal formulation by adding a nonlocal energy term to the misfit energy in the framework of original PN model for simplicity, and the nonlocal misfit energy is,

$$
N L E_{\text {misfit }}=E_{\text {misfit }}+E_{\text {nonlocal }}=\int \gamma[\delta(x)] d x+\iint \mathrm{K}\left(x-x^{\prime}\right) \delta(x) \delta\left(x^{\prime}\right) d x d x^{\prime}
$$


where $\Delta x$ is the atomic spacing in the slip plane perpendicular to the dislocation line, $a_{m}$ is the nonlocal coefficient, which can be computed by calibrating the nonlocal misfit energy $N L E_{\text {misfit }}$ against the atomistic simulations. Increasing $m$ corresponds to sampling the dislocation profiles farther and farther away from the local point at which the energy is being computed. The nonlocal effects decays quite rapidly, and nonlocal coefficients beyond $a_{5}$ are negligible [23]. Therefore, total dislocation energy of the nonlocal Peierls model is,

$$
\begin{aligned}
& E[\delta(x)]=E_{\text {elastic }}+E_{\text {misfit }}+E_{\text {nonlocal }}=K \iint \frac{d \delta\left(x^{\prime}\right)}{d x^{\prime}} \frac{\delta(x)}{x-x^{\prime}} d x d x^{\prime} \\
& +\int \gamma[\delta(x)] d x+\sum_{m=1}^{M} a_{m} \cdot \int \delta(x)\left(\delta(x)-\frac{\delta(x-m \Delta x)+\delta(x+m \Delta x)}{2}\right) d x
\end{aligned}
$$

Dislocation profile is then obtained by minimizing the total energy in Eq. (8). This nonlocal Peierls model often increases the slope of the dislocation profile, i.e., resulting in a narrow core of the dislocation, consequently, leading to a high Peierls stress. The discrete nature of the dislocation profile is not accounted for in this nonlocal formulations in Eq. (8). When the one-dimensional form is extended to multi-dimension, it is challenge in determining a series of nonlocal coefficients $a_{m}$.

\subsection{Improved nonlocal SVPN model}

To overcome the aforementioned shortcoming, here we propose new dislocation energy functions that incorporate the nonlocal atomic interactions corresponding to three-dimensional discrete discrete displacements along the disregistry profile.

\subsubsection{Dislocation energy functional}

The total energy for a dislocation is a functional of its disregistry vector $\vec{\delta}(x)$, 


$$
\begin{aligned}
& E_{\text {total }}[\vec{\delta}(x)]=E_{\text {elassic }}+E_{\text {misfit }}-E_{\text {app }}+E_{\text {nonlocal }}=\iint \frac{d \vec{\delta}\left(x^{\prime}\right)}{d x^{\prime}} \frac{H}{x-x^{\prime}} \vec{\delta}(x) d x d x^{\prime} \\
& +\int \gamma[\vec{\delta}(x)] d x-\sum_{k} \int \tau_{k} \cdot \delta_{k}(x) d x+\sum_{m=1}^{M} a_{m} \cdot \sum_{k} \int \delta_{k}(x)\left(\delta_{k}(x)-\frac{\delta_{k}(x-m \Delta x)+\delta_{k}(x+m \Delta x)}{2}\right) d x
\end{aligned}
$$

The first term corresponds to the elastic energy stored in the two half-spaces [10], the second term represents the atomic misfit energy in the glide plane [10], the third term describes the interaction of the applied stress $\tau_{k}$ with the dislocation profile $\delta_{k}[11,29]$, and the last term represents the nonlocal interaction energy of the atoms [23]. The first two energy terms are the same to those in the $\gamma$-PN model in Eq. (2), and the third term proposed in the SVPN model [11] in Eq. (3) to describe the interaction of the stress with the dislocation profile has been slightly modified as in Ref. [29], which can show the system instability more straightforwardly. The last nonlocal energy term is an extension of the original one dimensional form [23] by linearly adding the interaction along the $k$ direction. $a_{m}$ is the nonlocal coefficient, and increasing $m$ corresponds to incorporating the interactions further and further away from the local site. $\Delta x$ is the atomic spacing in the slip plane perpendicular to the dislocation line. $H$ is the anisotropic Stroh tensor $[25,26], \gamma[\vec{\delta}(x)]$ is the misfit potential, and $\tau_{k}$ is the applied stress, exactly the same meaning to those in the local PN models.

Considering the rapid decay of the nonlocal effects [23] far from the dislocation core, and $a_{1}$ (the near neighbor nonlocal interactions) is by far the most important contribution, we simplify the nonlocal energy term by setting the secondary nonlocal coefficients $a_{m}(m>1)$ to be zero, so the fourth nonlocal energy term in Eq. (9) is

$$
E_{\text {nonlocal }}\left[\vec{\delta}\left(x_{i}\right)\right]=a_{1} \sum_{k} \int \delta_{k}(x)\left(\delta_{k}(x)-\frac{\delta_{k}(x-\Delta x)+\delta_{k}(x+\Delta x)}{2}\right) d x
$$

Using the same discretization method in [11, 29], the disregistry profile is piecewise linearly interpolated between atomic sites $x_{i}\left(x_{i}=(i-N / 2) \cdot \Delta x\right.$, and the nonlocal energy is discretized as:

$$
E_{\text {nonlocal }}\left[\vec{\delta}\left(x_{i}\right)\right]=a_{1} \sum_{k} \sum_{i=1}^{N} \delta_{k}\left(x_{i}\right) \cdot\left(\delta_{k}\left(x_{i}\right)-\frac{\delta_{k}\left(x_{i+1}\right)+\delta_{k}\left(x_{i-1}\right)}{2}\right) \Delta x
$$

Replacing the nonlocal energy term in Eq. (9) with Eq. (11), we get the discretized energy functional for the 2D nonlocal SVPN model, 


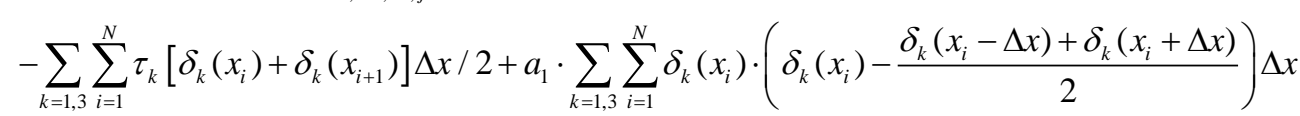

The discrete coefficient $\chi_{i j}$ is given in Eq. (4), and components of Stroh tensor $H_{i j}$ can be calculated from anisotropic elastic theory [25, 26]. $\rho_{k}\left(x_{i}\right)=\left[\delta_{k}\left(x_{i+1}\right)-\delta_{k}\left(x_{i}\right)\right] / \Delta x$ is the dislocation density in the $k$ direction. $N \cdot \Delta x$ is the cut-off distance in calculating the energy, and $\gamma\left[\delta_{1}\left(x_{i}\right), \delta_{3}\left(x_{i}\right)\right]$ is the 2D misfit potential.

Energy functional for the modified 2D nonlocal SVPN model in Eq. (12) can be extended to a 3D case, which can describe the dislocation core in full three dimensions, and account for the full relaxation of the dislocation profiles in three dimensions as the dislocation moves in lattice under the external stress. By incorporating the normal component of the disregistry vector $\delta_{2}$ in each energy term, we rewrote the energy function as

$$
\begin{aligned}
& E_{\text {disl }}\left[\left\{\delta_{1}\left(x_{i}\right), \delta_{2}\left(x_{i}\right), \delta_{3}\left(x_{i}\right)\right\}\right]=\sum_{k, l=1}^{3} \sum_{i, j=1}^{N} \rho_{k}\left(x_{i}\right) H_{k l} \rho_{l}\left(x_{j}\right) \chi_{i j}+\sum_{i=1}^{N} \gamma\left[\delta_{1}\left(x_{i}\right), \delta_{2}\left(x_{i}\right), \delta_{3}\left(x_{i}\right)\right] \Delta x \\
& -\sum_{k=1}^{3} \sum_{i=1}^{N} \tau_{k}\left[\delta_{k}\left(x_{i}\right)+\delta_{k}\left(x_{i+1}\right)\right] \Delta x / 2+a_{1} \cdot \sum_{k=1}^{3} \sum_{i=1}^{N} \delta_{k}\left(x_{i}\right) \cdot\left(\delta_{k}\left(x_{i}\right)-\frac{\delta_{k}\left(x_{i}-\Delta x\right)+\delta_{k}\left(x_{i}+\Delta x\right)}{2}\right) \Delta x
\end{aligned}
$$

where $\gamma\left[\delta_{1}\left(x_{i}\right), \delta_{2}\left(x_{i}\right), \delta_{3}\left(x_{i}\right)\right]$ is the 3D misfit potential, which will be explained in the next section. There is only one unknown parameter in the energy functional, the nonlocal coefficient $a_{1}$, which will be directly computed from the dislocation core structure. The dislocation profile at a certain external stress is obtained by minimizing the total energy in Eqs. (12) and (13). Peierls stress is determined as an instability of the energy function, i.e., the dislocation is moved evidently in crystal (on the order of several magnitude of Burgers vector [29-31]) above a critical resolved shear stress, while below which the dislocation profile only slightly adjusts its shape as a dislocation overcomes Peierls barrier, as will be presented in section 3.1 .

\subsubsection{Misfit potential $\gamma[\vec{\delta}]$}

Misfit potential describes the energy increase of the shear plane associated with the misfit vector. Corresponding to the three dimensional misfit vector, statics simulations using molecular statics simulations with embedded-atom-method potential [32, 33] or first 
principles calculations can be used to calculate the misfit potential [34-36].

Firstly, the 2D misfit potential is calculated similar to obtaining the gamma surface in Ref. [12]: (i) Cutting the crystal into two halves in the direction normal to the dislocation slip plane, (ii) rigidly shifting one half crystal with a misfit vector $\vec{u}=\left(u_{x}, u_{z}\right)$, (iii) calculating the energy increases per unit area caused by the misfit, in which the atoms are allowed to relax in the normal direction $y$, and the energy increases per unit area is the 2D misfit potential $\gamma\left[u_{x}\right.$, $u_{z}$ ].

Secondly, a 3D misfit potential is calculated similar to the 2D case. The only difference is the misfit vector is three dimensional $\vec{u}=\left(u_{x}, u_{y}, u_{z}\right)$, and the atoms are fixed in three directions when calculating the energy difference caused by the misfit. By discretely sampling the misfit vector $\vec{u}$ from $\vec{\delta}_{\min }$ to $\vec{\delta}_{\max }$ with increment $\Delta u=0.0015 \AA$, and repeating calculating the energy increase caused by the misfit, the discrete $2 \mathrm{D}$ or $3 \mathrm{D}$ misfit potential $\gamma[\vec{u}]$ is obtained.

Finally, to get the gamma value at any disregistry, i.e., $\gamma\left[\delta_{1}\left(x_{i}\right), \delta_{3}\left(x_{i}\right)\right]$ in Eq. (12) or $\gamma\left[\delta_{1}\left(x_{i}\right), \delta_{2}\left(x_{i}\right), \delta_{3}\left(x_{i}\right)\right]$ in Eq. (13), serendipity shape functions [37] are used to interpolate between the discrete misfit potential $\gamma[\vec{u}]$ calculated from molecular statics simulations.

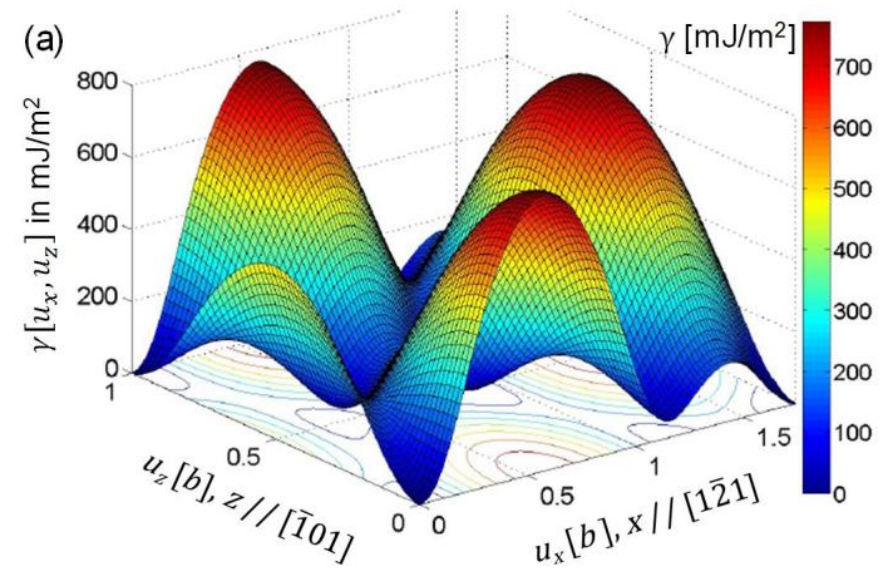




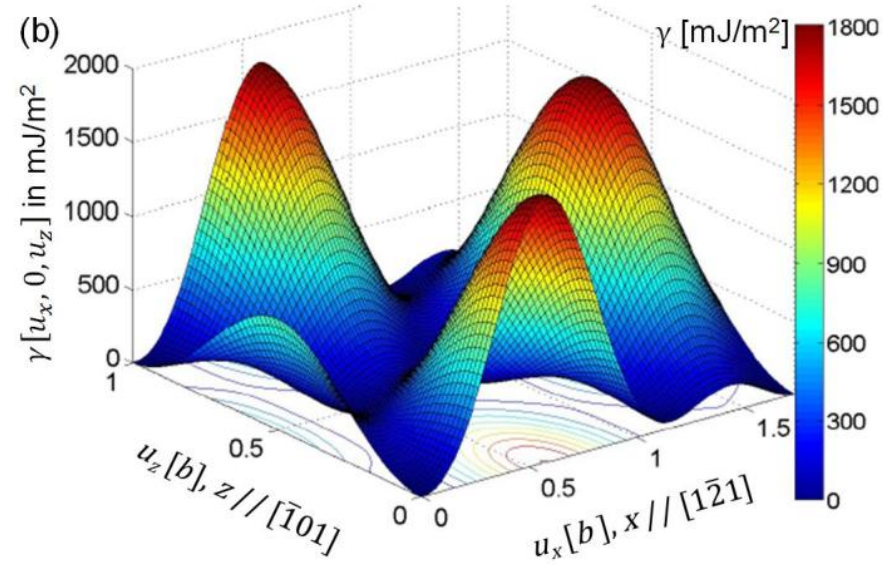

Fig. 1 Misfit potential for (111) plane of copper calculated by molecular statics simulations. (a) Atoms are allowed to relax in the normal direction during the energy minimization after the shear. (b) Normal misfit $u_{y}$ is fixed to be zero.

Misfit potential for the (111) plane of copper calculated by molecular statics simulation is compared in Fig. 1, where Fig. 1a is the 2D misfit potential, and Fig. 1b is the 3D misfit potential when the normal misfit $u_{y}$ is fixed to be zero. Obviously, when the atomic relaxation is allowed in the normal direction $y$, the unstable stacking fault energy is significantly reduced.

\subsubsection{Solving procedure}

We take the typical dislocations in copper and iron as model systems. Table 1 lists the Burgers vector, dislocation line, the slip plane, and the slip direction. All the dislocations are characterized by a disregistry vector $\vec{\delta}$, defined as the relative displacement of the atom pairs across the slip plane. It generally has three components $\delta_{k}, k=1,2,3$ denotes the disregistry profile or dislocation profile is along the $x$-, $y$-, and $z$ - direction, respectively. Note the disregistry vector can be either $\vec{\delta}(x)$ or $\vec{\delta}(z)$, depending on the slip direction being $x$ or $z$. They are all denoted as $\vec{\delta}(x)$ in the energy functional just for simplicity. Adopting the coordinates in Table 1 for the dislocations studied in this work, the components of the Stroh tensor $H_{k j}$ calculated according to the anisotropic elastic theory $[25,26]$ are shown in Table 2, and the elastic constants calculated by molecular statics simulations using embedded-atom-method potential $[32,33]$ are also shown in Table 2. 
Table 1. Geometries and coordinates setup for $a / 2<110>\{111\}$ screw and edge dislocations in copper and an $a / 2<111>\{110\}$ edge dislocation in iron.

\begin{tabular}{ccccccc}
\hline & & Burger vector & Dislocation line & Slip plane & Slip direction & $\Delta x$ \\
\hline \multirow{2}{*}{$\mathrm{Cu}$} & screw & $a / 2[\overline{1} 01], / / z$ & {$[\overline{1} 01], / / z$} & $(111), \perp y$ & {$[1 \overline{2} 1], / / x$} & $\sqrt{3} b / 2=2.214 \AA$ \\
& edge & $a / 2[\overline{1} 01], / / z$ & {$[1 \overline{2} 1], / / x$} & $(111), \perp y$ & {$[\overline{1} 01], / / z$} & $b=2.556 \AA$ \\
$\mathrm{Fe}$ & edge & $a / 2[111], / / x$ & {$[11 \overline{2}], / / z$} & $(1 \overline{1} 0), \perp y$ & {$[111], / / x$} & $b=2.4825 \AA$ \\
\hline
\end{tabular}

Table 2. Components of the Stroh tensor for the dislocations in $\mathrm{Cu}$ and Fe.

\begin{tabular}{ccccccccccccc}
\hline & \multicolumn{9}{c}{$H_{k l}$ (in GPa) } & \multicolumn{6}{c}{ Elastic constants (in GPa) } \\
& & $H_{11}$ & $H_{22}$ & $H_{33}$ & $H_{12}$ & $H_{13}$ & $H_{23}$ & $c_{11}$ & $c_{12}$ & $c_{44}$ & $\mu$ \\
\hline \multirow{2}{*}{$\mathrm{Cu}$} & screw & 6.0517 & 6.3294 & 3.3774 & -0.3927 & 0 & 0 & 169.9 & 122.6 & 76.2 & 41.2 \\
& edge & 3.5492 & 6.0114 & 5.9441 & 0.6959 & 0 & 0 & & & & \\
\multirow{2}{*}{$\mathrm{Fe}$} & edge & 9.6839 & 9.437 & 6.1481 & 0 & -0.8986 & 0 & 243.4 & 145.1 & 116.0 & 71.5 \\
\hline
\end{tabular}

(1) Initialization, boundary conditions and external stress

Volterra dislocation was taken as the initial trial solution for the dislocation profiles. That is $\delta_{k}\left(x_{i}\right)=0$ for the lower half $x_{i}$ and $\delta_{k}\left(x_{i}\right)=b_{k}$ for the upper half $x_{i}$. For example, for the edge dislocation in $\mathrm{Cu}$, only $b_{3}=b$, the other two components should be zero $b_{1}=b_{2}=0$, so the disregistry vector was initialized as,

$$
\begin{aligned}
\delta_{3}\left(x_{i}\right) & = \begin{cases}0, & i \leq N / 2 \\
b, & i>N / 2\end{cases} \\
\delta_{1}\left(x_{i}\right) & =\delta_{2}\left(x_{i}\right)=0, \quad 1 \leq i \leq N
\end{aligned}
$$

The dislocation profiles $\delta_{k}$ at the two ends $x_{i}(i=1$ or $N)$ were fixed, while their values at all the other atomic sites in between the two ends $x_{i}(2 \leq i \leq N-1)$ were allowed to vary in the energy minimization process so as to obtain the equilibrium dislocation core structure. The cut-off distance $N \cdot \Delta x$ in the energy functional are set to be $200 \Delta x$ and $100 \Delta x$ for dislocations in $\mathrm{Cu}$ and $\mathrm{Fe}$ respectively, according to the extended or compact core. The external stress was applied to the dislocation by increasing $\tau_{k}$ in the energy functional. To make the resolved shear stress equal to the applied shear stress for simplicity, only $\tau_{y z}\left(=\tau_{3}\right)$ was applied to the dislocations in $\mathrm{Cu}$, and only $\tau_{y x}\left(=\tau_{1}\right)$ was applied to the edge dislocation in Fe.

(2) Nonlocal coefficient

The nonlocal coefficient $a_{1}$ is determined in two steps: (i) The disregistry profile at any 
$a_{1}$ and zero applied stress is firstly calculated by minimizing the total energy in Eqs. (12) and (13) with a simple steepest descent method. (ii) The optimal $a_{1}$ is determined to minimize the sum of the squared errors of the disregistry profile between the prediction of nonlocal SVPN model and the corresponding MD simulations (details of MD simulations can be found in the supplementary materials of Ref. [30]):

$$
\begin{aligned}
& \text { Dislocations in Cu: } a_{1}=\arg \min \left\{\sum_{i}\left[\delta_{3}^{a_{1}}\left(x_{i}\right)-\delta_{3}^{M D}\left(x_{i}\right)\right]^{2}\right\} \\
& \text { edge in Fe: } a_{1}=\arg \min \left\{\sum_{i}\left[\delta_{1}^{a_{1}}\left(x_{i}\right)-\delta_{1}^{M D}\left(x_{i}\right)\right]^{2}\right\}
\end{aligned}
$$

For the screw and edge dislocations in $\mathrm{Cu}$, the nonlocal coefficient $a_{1}$ determined by Eq. (15) is $0.575 \mu / b$ and $0.905 \mu / b$ respectively, $\mu$ being the shear modulus in the $\{111\}$ plane along the $<110>$ direction (see Table 2). And for the edge dislocation in Fe, $a_{1}$ is $1.396 \mu / b, \mu$ being the shear modulus in the $\{110\}$ plane along the $<111>$ direction.

(3) Core structure and Peierls stress

Equilibrium core structure was obtained by minimizing the total energy functional in Eq. (12) or (13) with steepest descent method. Peierls stress was determined from the energy functional in two steps. Firstly, the external stress was gradually applied to dislocations in Eq. (12) or (13), and the equilibrium disregistry profiles for each certain external stress was obtained by minimizing the total energy. Secondly, we search for the critical resolved shear stress when the system instability happens, indicated by significant movement of the dislocation (see Fig. 4), and this critical stress is determined as Peierls stress [29-31].

\section{Results and discussion}

\subsection{Core structure}

Core structure of the dislocations predicted by the 2D and 3D models are compared from the three components of the disregistry vector. Fig. 2 shows the dislocation profiles at zero stress for the edge dislocation in $\mathrm{Cu}$. Clearly, the 3D nonlocal SVPN model can predict almost the same the dislocation profiles with MD simulations. While dislocation profiles predicted by the 3D and 2D local SVPN model ( $a_{1}=0$, without the nonlocal energy term) 

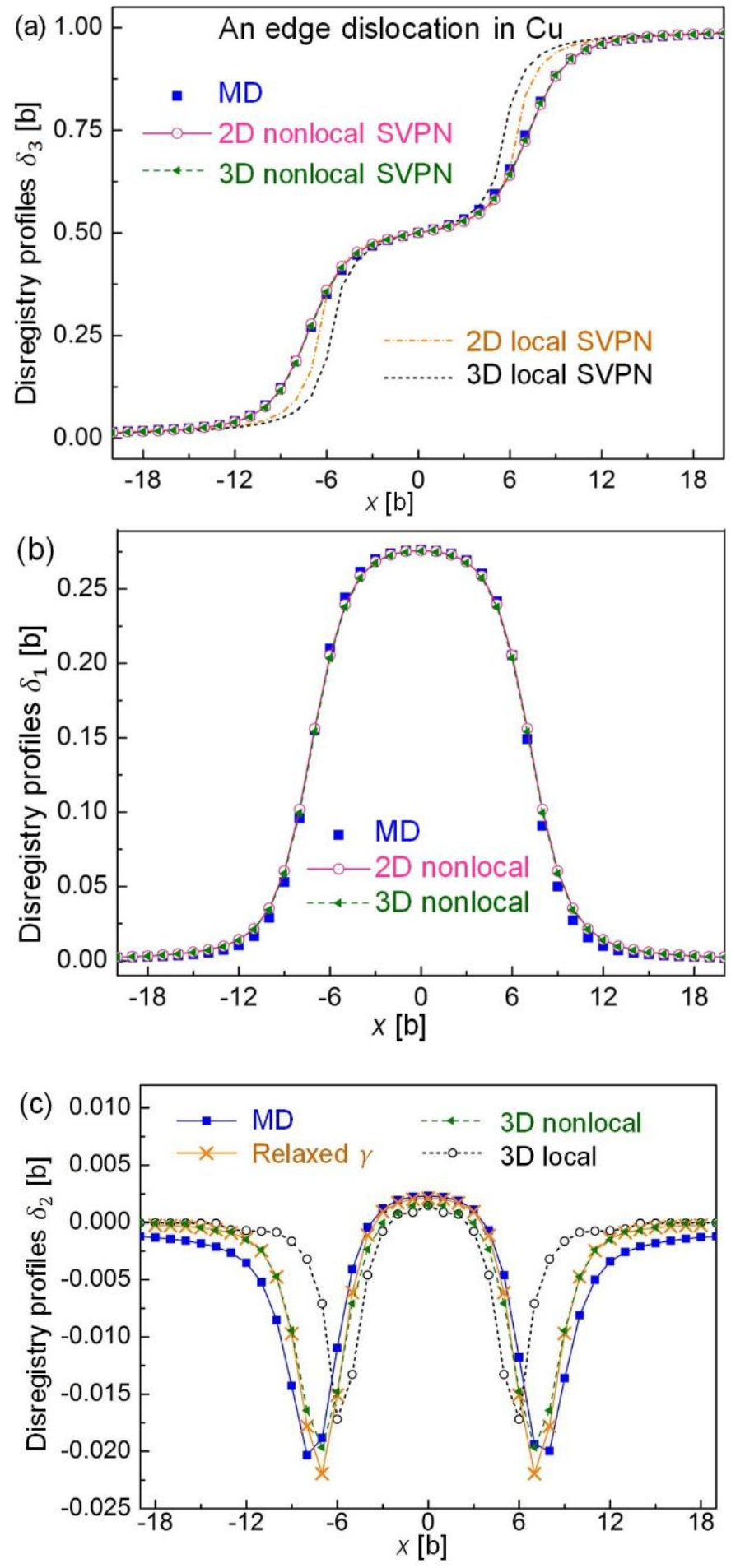

Fig. 2 Disregistry profiles for an edge dislocation in copper at zero stress predicted by the 2D and 3D SVPN models, and MD simulations: (a) edge component $\delta_{3}$, (b) screw component $\delta_{1}$ and (c) normal component $\delta_{2}$.

The good predictions of the core structure by our 2D and 3D nonlocal SVPN models are also found for the screw dislocation in $\mathrm{Cu}$ and edge dislocation in Fe. Fig. 3 shows the 

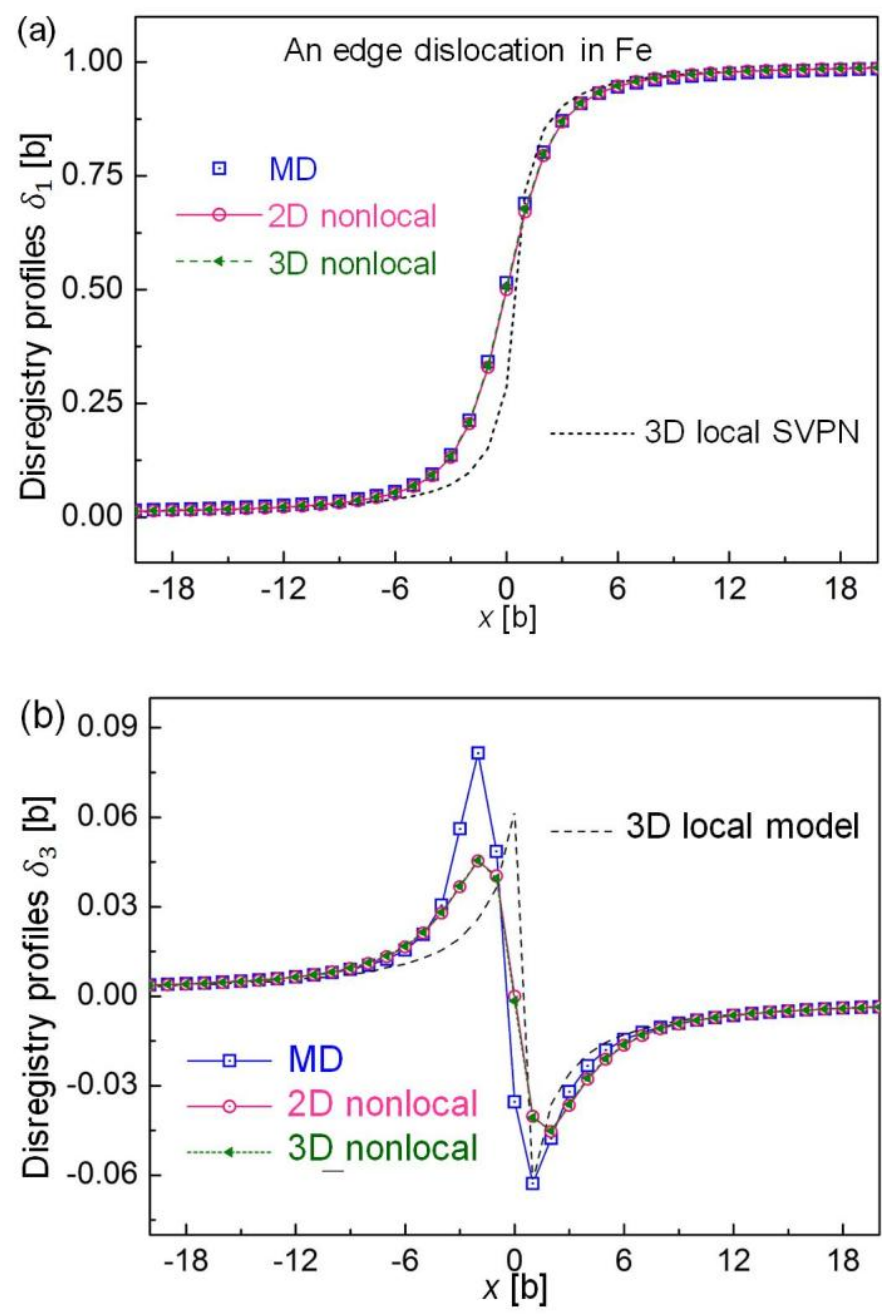


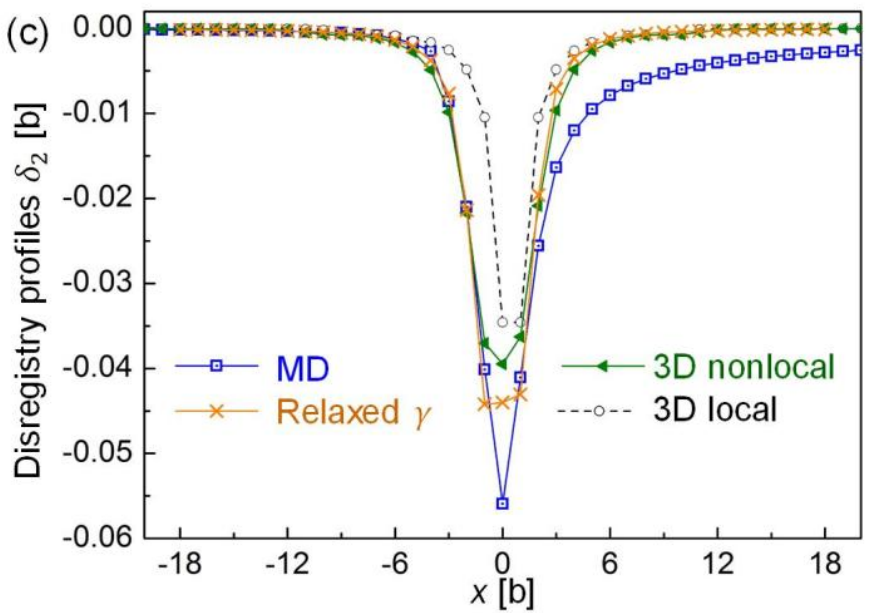

Fig. 3 Disregistry profiles for an edge dislocation in iron at zero stress predicted by the 2D and 3D SVPN models, and MD simulations: (a) edge component $\delta_{1}$, (b) screw component $\delta_{3}$ and (c) normal component $\delta_{2}$.

To more transparently present how the dislocation core structure is improved by the nonlocal energy term, dislocation width $(w)$ computed by different models is compared in Table 3. For the local/nonlocal SVPN model and MD calculations, $w$ is directly computed from the main component of the disregistry vector, defined as the distance between where $\delta_{3}$ (for the dislocations in $\mathrm{Cu}$ ) or $\delta_{1}$ (for the edge dislocation in $\mathrm{Fe}$ ) equals to $0.75 b$ and $0.25 b$, respectively. Table 3 shows that, for all the dislocations considered in this work, the nonlocal SVPN models (both 2D and 3D ones) predict wider dislocation core than the local SVPN models, and the nonlocal model predictions of dislocation width are closer to either experiments finding [39] or ab initio calculations[40]. Besides, the 2D nonlocal SVPN model can predict almost the same dislocation width with the 3D nonlocal SVPN model.

Table 3. Comparison of dislocation width $w$ (in units of $b$ ) for dislocations in $\mathrm{Cu}$ and $\mathrm{Fe}$.

\begin{tabular}{cccccccc}
\hline & \multicolumn{5}{c}{ Dislocation width $w$ (in $b)$} \\
\cline { 3 - 8 } & & 2D local & 3D local & 2D nonlocal & 3D nonlocal & MD & Reference \\
\hline \multirow{2}{*}{$\mathrm{Cu}$} & screw & 3.7 & 3.7 & 5.5 & 5.6 & 5.5 & $7.0[39]$ \\
& edge & 13.1 & 11.4 & 14.6 & 14.5 & 14.4 & $14.9[39]$ \\
\multirow{2}{*}{$\mathrm{Fe}$} & edge & 1.6 & 1.5 & 3.3 & 3.3 & 3.2 & $3.0[40]$ \\
\hline
\end{tabular}

\subsection{Peierls stress}

Peierls stress is determined as the critical resolved shear stress to move the dislocation. 

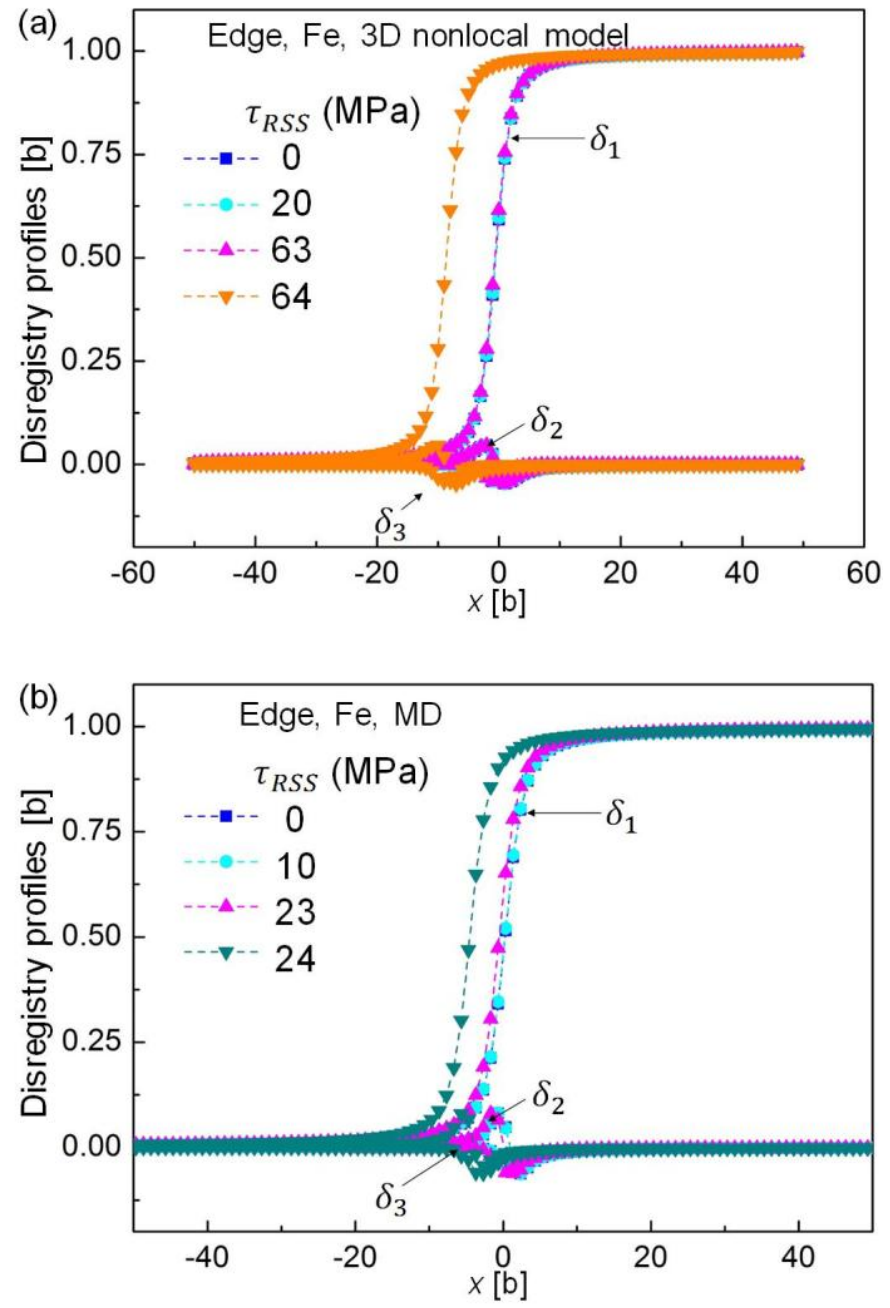

Fig. 4 Response of the edge dislocation profiles in iron to increasing resolved shear stress calculated by (a) 3D nonlocal SVPN model and (b) MD simulations. Peierls stress, determined as the critical stress to move the mixed dislocation, is $63 \mathrm{MPa}$ and $23 \mathrm{MPa}$ respectively.

Comparison of the Peierls stress predicted by different models are shown in Table 4, which indicates that: (1) The 2D nonlocal SVPN model can predict almost the same Peierls stress as the 3D nonlocal SVPN model, as expected from the analysis of the core structure in section 3.1. (2) Compared with MD predictions, the nonlocal SVPN models considerably 
improves the prediction of the Peierls stress than the local SVPN model. For the dislocations in $\mathrm{Cu}$, Peierls stress by the nonlocal models is in the same order of the MD predictions and experimental estimation ( 1 MPa) [3]. For the edge dislocation in Fe, Peierls stress by the nonlocal models is also in the same order of MD predictions, even though three times higher than MD predictions, while the local model predictions are orders of magnitude higher than MD predictions [41]. So the prediction of Peierls stress is much improved by the nonlocal SVPN model than the local SVPN models.

Table 4. Comparison of Peierls stress for dislocations in copper and iron calculated by the three dimensional (3D) and two dimensional (2D) nonlocal SVPN model, and MD simulations.

\begin{tabular}{ccccccc}
\hline & & \multicolumn{5}{c}{ Peierls stress $\tau_{p}($ in MPa) } \\
\cline { 3 - 7 } & & MD & 3D nonlocal & 2D nonlocal & 2D local & 3D local \\
\hline \multirow{2}{*}{$\mathrm{Cu}$} & screw & 2.9 & 5 & 5 & 119 & 145 \\
& edge & 1.3 & 4 & 3 & 72 & 80 \\
$\mathrm{Fe}$ & edge & 23 & 63 & 89 & 1019 & 1050 \\
\hline
\end{tabular}

\section{Conclusions}

Previous local and nonlocal PN models are critically reviewed, and then an improved nonlocal SVPN model is developed by accounting for the nonlocal atomic interactions in the semi-discrete variational Peierls framework. By directly computing the nonlocal coefficient based on the core structure obtained by MD simulations, the 2D and 3D nonlocal SVPN model significantly improves the prediction ability of the dislocation core structure and Peierls stress than the local PN models.

Compared with previous local and nonlocal PN models, the nonlocal SVPN model developed in this work appears to be a more reliable one, not only for the good predictions of dislocation profile and Peierls stress presented in section 3, but also for the much simpler form of the nonlocal energy term and the easier but more physically realistic way to determine the unique nonlocal coefficient from dislocation core structure. This is of special value to apply the nonlocal SVPN model to predict Peierls stress, especially for dislocations with complex core structures, because elastic constants and misfit potential can be accurately 
obtained by combing experiments [42] and ab initio calculations [35, 36], and the dislocation dependent nonlocal coefficient can be directly computed from core structure, which can be acquired by experiment techniques $[43,44]$. Combing these advantages, the nonlocal SVPN model can be applied or generalized to study core structure and Peierls stress for dislocations in bilayer materials [45, 46] and grain boundaries [20, 47], and also for the curved dislocations [18, 48].

Last but not least, the 2D nonlocal SVPN model with relaxed 2D misfit potential as input for dislocation misfit energy can predict almost the same results as the $3 \mathrm{D}$ nonlocal SVPN model. This finding validates the 2D nonlocal SVPN model and implies that the 2D misfit potential by allowing for the atomic relaxation in the normal direction can effectively describe the three dimensional interatomic interactions in the slip plane, and therefore can reduce the computation cost for the full 3D misfit potential.

\section{Acknowledgement}

This research was supported by the National Science Foundation of China project Grant No. 51471107 and 51671132, and CCS project Grant No. YK 2015-0202002, and Center for High Performance Computing, Shanghai Jiao Tong University.

\section{References}

[1] F. Nabarro. Fifty-year study of the Peierls-Nabarro stress, Mater. Sci. Eng. A 234 (1997) 67-76.

[2] F. Nabarro. Theoretical and experimental estimates of the Peierls stress, Philos. Mag. A 75 (1997) 703-711.

[3] Y. Kamimura, K. Edagawa, S. Takeuchi. Experimental evaluation of the Peierls stresses in a variety of crystals and their relation to the crystal structure, Acta Mater. 61 (2013) 294-309.

[4] S.K. Yadav, R. Ramprasad, A. Misra, X.Y. Liu. Core structure and Peierls stress of edge and screw dislocations in TiN: A density functional theory study, Acta Mater. 74 (2014) 268-277.

[5] Z. Wu, W. Curtin. The origins of high hardening and low ductility in magnesium, Nature $526(2015) 62-67$. 
[6] Z.X. Wu, W.A. Curtin. Mechanism and energetics of < c plus a > dislocation cross-slip in hcp metals, Proc. Natl. Acad. Sci. U.S.A. 113 (2016) 11137-11142.

[7] I. Belabbas, J. Chen, M.I. Heggie, C.D. Latham, M.J. Rayson, P.R. Briddon, G. Nouet. Core properties and mobility of the basal screw dislocation in wurtzite GaN: a density functional theory study, Model. Simul. Mater. Sci. Eng. 24 (2016) 075001.

[8] R. Peierls. The size of a dislocation, Proc. Physs. Soc. 52 (1940) 34-37.

[9] F. Nabarro. Dislocations in a simple cubic lattice, Proc. Physs. Soc. 59 (1947) 256.

[10] G. Schoeck. The Peierls model: progress and limitations, Mater. Sci. Eng. A 400 (2005) 7-17.

[11] V.V. Bulatov, E. Kaxiras. Semidiscrete variational Peierls framework for dislocation core properties, Phys. Rev. Lett. 78 (1997) 4221.

[12] V. Vitek. Intrinsic stacking faults in body-centred cubic crystals, Philos. Mag. 18 (1968) 773-786.

[13] V. Vitek, F. Kroupa. Generalized splitting of dislocations, Philos. Mag. 19 (1969) 265-284.

[14] G. Schoeck. The generalized Peierls-Nabarro model, Philos. Mag. A 69 (1994) 1085-1095.

[15] G. Schoeck. The Peierls dislocation: Line energy, line tension, dissociation and deviation, Acta Mater. 45 (1997) 2597-2605.

[16] B. Joos, M. Duesbery. The Peierls stress of dislocations: an analytic formula, Phys. Rev. Lett. 78 (1997) 266-269.

[17] B. Joos, Q. Ren, M. Duesbery. Peierls-Nabarro model of dislocations in silicon with generalized stacking-fault restoring forces, Phys. Rev. B 50 (1994) 5890.

[18] Y. Xiang, H. Wei, P. Ming, E. Weinan. A generalized Peierls-Nabarro model for curved dislocations and core structures of dislocation loops in $\mathrm{Al}$ and $\mathrm{Cu}$, Acta Mater. 56 (2008) 1447-1460.

[19] S. Dai, Y. Xiang, D.J. Srolovitz. Atomistic, generalized Peierls-Nabarro and analytical models for (lll 11 1) twist boundaries in $\mathrm{Al}, \mathrm{Cu}$ and $\mathrm{Ni}$ for all twist angles, Acta Mater. 69 (2014) 162-174.

[20] C. Shen, J. Li, Y. Wang. Predicting structure and energy of dislocations and grain boundaries, Acta Mater. 74 (2014) 125-131.

[21] L. Liu, X. Wu, R. Wang, H. Feng, S. Wu. High-pressure effect on elastic constants, stacking fault energy and correlation with dislocation properties in $\mathrm{MgO}$ and $\mathrm{CaO}$, Eur. Phys. J. B 85 (2012) 1-10.

[22] G. Schoeck. Peierls energy of dislocations: a critical assessment, Phys. Rev. Lett. 82 
(1999) 2310.

[23] R. Miller, R. Phillips, G. Beltz, M. Ortiz. A non-local formulation of the Peierls dislocation model, J. Mech. Phys. Solids 46 (1998) 1845-1867.

[24] V. Bulatov, W. Cai. Computer simulations of dislocations, Oxford University Press, 2006.

[25] D. Bacon, D. Barnett, R.O. Scattergood. Anisotropic continuum theory of lattice defects, Prog. Mater. Sci. 23 (1980) 51-262.

[26] J. Hirth, J. Lothe. Theory of Dislocations, second ed., Wiley, New York, 1982.

[27] G. Lu, V.V. Bulatov, N. Kioussis*. A nonplanar Peierls-Nabarro model and its application to dislocation cross-slip, Philos. Mag. 83 (2003) 3539-3548.

[28] G. Lu, V.V. Bulatov, N. Kioussis. On stress assisted dislocation constriction and cross-slip, Int. J. Plast. 20 (2004) 447-458.

[29] Y. Shen, X. Cheng. Dislocation movement over the Peierls barrier in the semi-discrete variational Peierls framework, Scripta Mater. 61 (2009) 457-460.

[30] G. Liu, X. Cheng, J. Wang, K. Chen, Y. Shen. Peierls stress in face-centered-cubic metals predicted from an improved semi-discrete variation Peierls-Nabarro model, Scripta Mater. 120 (2016) 94-97.

[31] X. Cheng, Y. Shen, L. Zhang, X. Liu. Surface effect on the screw dislocation mobility over the Peierls barrier, Philos. Mag. Lett. 92 (2012) 270-277.

[32] G.J. Ackland, D.J. Bacon, A.F. Calder, T. Harry. Computer simulation of point defect properties in dilute $\mathrm{Fe}-\mathrm{Cu}$ alloy using a many-body interatomic potential, Philos. Mag. A 75 (1997) 713-732.

[33] Y. Mishin, M. Mehl, D. Papaconstantopoulos, A. Voter, J. Kress. Structural stability and lattice defects in copper: Ab initio, tight-binding, and embedded-atom calculations, Phys Rev. B 63 (2001) 224106.

[34] Y.-M. Juan, E. Kaxiras. Generalized stacking fault energy surfaces and dislocation properties of silicon: A first-principles theoretical study, Philos. Mag. A 74 (1996) 1367-1384.

[35] G. Lu, N. Kioussis, V.V. Bulatov, E. Kaxiras. Generalized-stacking-fault energy surface and dislocation properties of aluminum, Phys. Rev. B 62 (2000) 3099.

[36] L. Liu, X. Wu, R. Wang, W. Li, Q. Liu. First principle study on the temperature dependent elastic constants, anisotropy, generalized stacking fault energy and dislocation core of NiAl and FeAl, Comput. Mater. Sci. 103 (2015) 116-125.

[37] R.L. Taylor. On completeness of shape functions for finite element analysis, Int. J. Numer. Meth. Eng. 4 (1972) 17-22. 
[38] M. Yamaguchi, V. Vitek. Core structure of nonscrew 1/2 (111) dislocations on (110) planes in bcc crystals. I. Core structure in an unstressed crystal, J. Phys. F 3 (1973) 523.

[39] W. Stobbs, C. Sworn. The weak beam technique as applied to the determination of the stacking-fault energy of copper, Philos. Mag. 24 (1971) 1365-1381.

[40] J.-A. Yan, C.-Y. Wang, S.-Y. Wang. Generalized-stacking-fault energy and dislocation properties in Itextbf\{ Itextit\{bcc\} \} Fe: A first-principles study, Phys. Rev. B 70 (2004) 174105.

[41] Y.N. Osetsky, D.J. Bacon. An atomic-level model for studying the dynamics of edge dislocations in metals, Model. Simul. Mater. Sci. Eng. 11 (2003) 427.

[42] H.B. Huntington. The elastic constants of crystals, Solid State Phys. 7 (1958) 213-351.

[43] M.J. Mills, N.L. Baluc, P.M. Sarosi. HRTEM of dislocation cores and thin-foil effects in metals and intermetallic compounds, Microsc. Res. Tech. 69 (2006) 317-329.

[44] C.W. Zhao, Y.M. Xing, P.C. Bai. Experimental examination of displacement field in an edge dislocation core in aluminum, Phys. Lett. A 372 (2008) 312-315.

[45] S. Dai, Y. Xiang, D.J. Srolovitz. Twisted Bilayer Graphene: Moiré with a Twist, Nano Lett. 16 (2016) 5923-5927.

[46] S. Dai, Y. Xiang, D.J. Srolovitz. Structure and energetics of interlayer dislocations in bilayer graphene, Phys. Rev. B 93 (2016) 085410.

[47] S. Dai, Y. Xiang, D.J. Srolovitz. Structure and energy of $\left(\begin{array}{lll}1 & 1 & 1\end{array}\right)$ low-angle twist boundaries in $\mathrm{Al}, \mathrm{Cu}$ and $\mathrm{Ni}$, Acta Mater. 61 (2013) 1327-1337.

[48] H. Wei, Y. Xiang. A generalized Peierls-Nabarro model for kinked dislocations, Philos. Mag. 89 (2009) 2333-2354. 


\section{Original PN model}

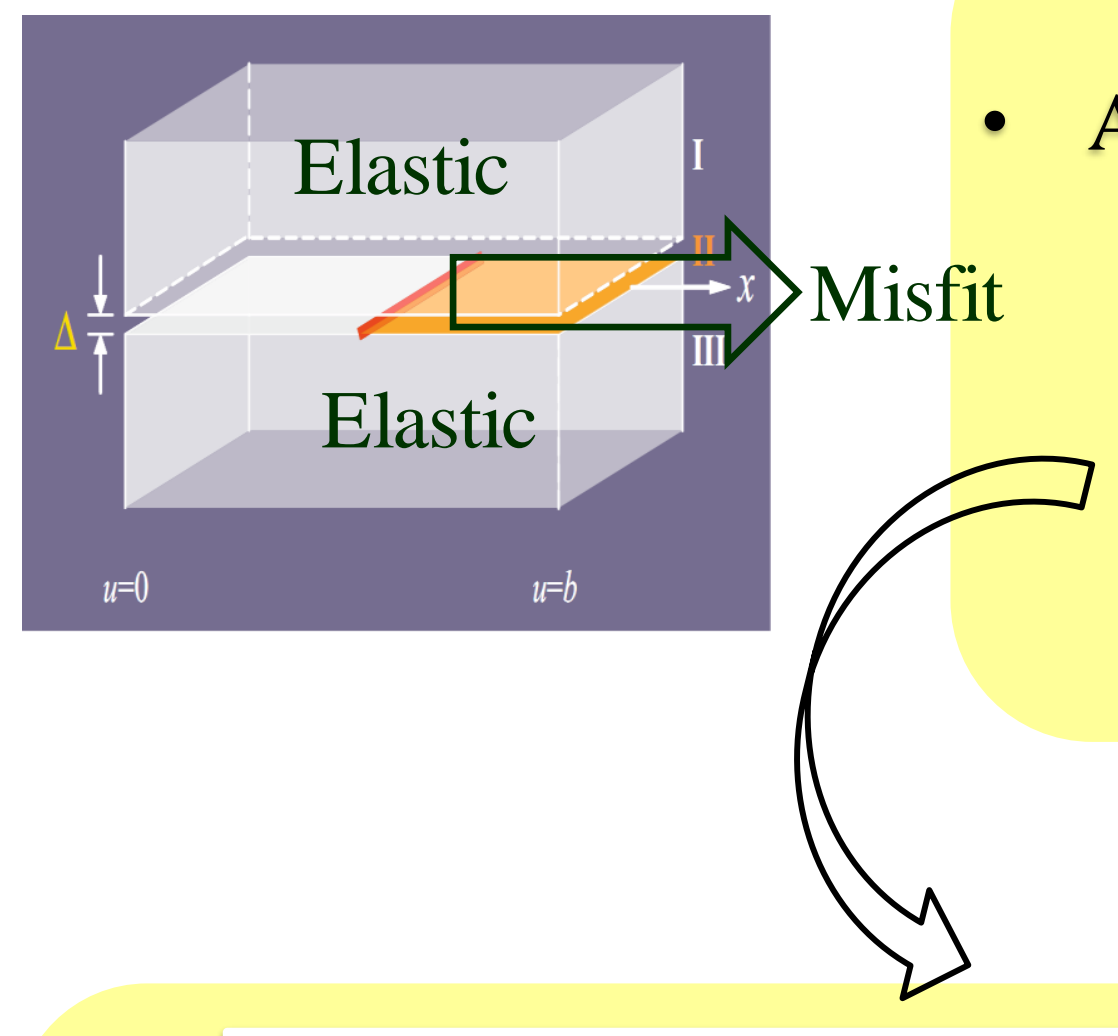

To address \# 1. $\gamma$-PN model

- $2 \mathrm{D}$ atomic force $=-\nabla \gamma[\vec{\delta}]$

To address \# 1-3. SVPN model

- Discrete summation of 3D $\gamma[\vec{\delta}]$

- Considering applied stress

Analytical solution for dislocation profile and Peierls stress

Shortcomings:

1. 1D sinusoidal atomic restoring force

2. Inconsistency in calculating misfit energy

3. Rigid translation of dislocation profile

4. Ignorance of nonlocal atomic interaction

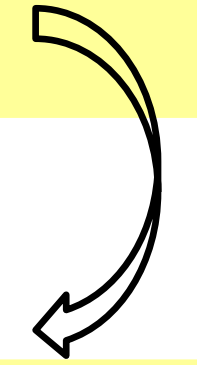

To address \# 4. Nonlocal PN model

a. 1D nonlocal Peierls model: Original PN + nonlocal energy $E_{\text {nonlocal }}=\iint K\left(x-x^{\prime}\right) \delta(x) \delta\left(x^{\prime}\right) d x d x^{\prime}$

b. 2D nonlocal $\gamma$-PN model: nonlocal misfit energy

$$
E_{\text {misfit }}=\sum_{n=1}^{N} \Gamma\left[x_{n}\right] \Delta x, \Gamma\left[x_{n}\right]=\frac{1}{2 t} \int_{-t}^{t} \gamma[\vec{\delta}(x+t)] d t
$$

- SVPN + nearest neighbor nonlocal atomic interaction (simpler)

- Calibrate core structure to obtain nonlocal coefficient

- Improve core structure and Peierls stress 